\title{
CONTINUOUS SETS THAT HAVE NO CONTINUOUS SETS OF CONDENSATION.
}

BY PROFESSOR R. L. MOORE.

(Read before the American Mathematical Society, October 27, 1917.).

JANISZEwski has shown* that if $A$ and $B$ are two distinct points then every bounded set of points that is irreducibly continuous $\dagger$ from $A$ to $B$, and has no continuous set of condensation, $\dagger$ is a simple continuous arc from $A$ to $B$. In the present paper I will establish the following result.

Theorem. Every bounded continuous set of points that has no continuous set of condensation is a continuous curve. $\ddagger$

Proof. Suppose $M$ is a bounded continuous set of points that has no continuous set of condensation. It has been shown by Hahn§ that every bounded continuous set of points that is connected "im kleinen" is a continuous curve. I shall proceed to show that the point set $M$ is connected "im kleinen." Suppose that it is not. Then there is a point $P$ belonging to $M$ and a circle $K$ with center at $P$ such that within every circle whose center is $P$ there exists a point which does not lie together with $P$ in any connected subset of $M$ that lies

* S. Janiszewski, "Sur les continus irréductibles entre deux points," Journal de l'Ecole Polytechnique, 2e série, vol. 16 (1911-12), pp. 79-170.

$\dagger \mathrm{A}$ set of points is said to be connected if, however it be divided into two mutually exclusive subsets, one of these subsets contains a limit point of the other one. A set of points is said to be continuous if it is closed and connected and contains more than one point. A continuous set of points containing the two distinct points $A$ and $B$ is said to be irreducibly continuous from $A$ to $B$ if it contains no other continuous set that contains both $A$ and $B$. The continuous set $N$ is said to be a continuous set of condensation of the continuous set $M$ if $N$ is a subset of $M$ and every point of $N$ is a limit point of $M-N$.

$\ddagger$ A continuous curve is the set of all points $\{(x, y)\}$ satisfying the equations $x=f_{1}(t), y=f_{2}(t)(0 \leqq t \leqq 1)$, where $f_{1}(t)$ and $f_{2}(t)$ are continuous functions of $t$. In case there do not exist, in the interval $(0 \leqq t \leqq 1)$, two distinct numbers $t_{1}$ and $t_{2}$ such that $f_{1}\left(t_{1}\right)=f_{1}\left(t_{2}\right)$ and $f_{2}\left(t_{1}\right)=f_{2}\left(t_{2}\right)$, then this curve is a simple continuous arc.

$\S$ Hans Hahn, "Ueber die allgemeinste ebene Punktmenge, die stetiges Bild einer Strecke ist," Jahresbericht der Deutschen Mathematiker-Vereinigung, vol. 23 (1914), pp. 318-322. A set of points $M$ is said to be connected "im kleinen" (cf. Hahn, loc. cit.) if for each point $P$ of $M$ and each circle $K$ with center at $P$ there exists, within $K$, another circle $K^{\prime}$, with center at $P$, such that if $X$ is a point of $M$ within $K^{\prime}$ then $X$ and $P$ lie together in some connected subset of $M$ that lies entirely within $K$. 
entirely within $K$. Let $K_{1}, K_{2}, K_{3}, \cdots$ denote an infinite sequence of circles with center at $P$ such that the radius of $K_{n}$ is $r / 2 n$, where $r$ is the radius of $K$. For every $n$ there exists, within $K_{n}$, a point $X_{n}$ such that $X_{n}$ and $P$ do not lie together in any connected subset of $M$ that lies entirely within $K$. Let $\bar{K}$ denote a definite circle, with center at $P$, that lies within $K$ and encloses $K_{1}$. By a theorem due to Janiszewski,* $M$ contains at least one point set that is irreducibly continuous from $X_{n}$ to $P$. Let $t_{n}$ denote one such set. The set $t_{n}$ is a subset of $M$ and $M$ has no continuous set of condensation. It follows that $t_{n}$ has no continuous set of condensation. Hence, by the first of the above mentioned theorems of Janiszewski, $t_{n}$ is a simple continuous arc $\dagger$ from $X_{n}$ to $P$. This arc can not lie entirely within $K$. Let $Y_{n}$ denote the first point, in the order from $X_{n}$ to $P$, that it has in common with $\bar{K}$. The interval $X_{n} Y_{n}$ of the arc $t_{n}$ lies, except for the point $Y_{n}$, entirely within $\bar{K}$. There exists $n_{1}$ such that if $n \geqq n_{1}$ then $X_{n} Y_{n}$ has no point in common with $X_{1} Y_{1}$. For otherwise there would exist an infinite subsequence $\overline{X_{1}} \overline{Y_{1}}$, $\bar{X}_{2} \bar{Y}_{2}, \bar{X}_{3} \bar{Y}_{3}, \cdots$ of the sequence $X_{1} Y_{1}, X_{2} Y_{2}, X_{3} Y_{3}, \cdots$ such that, for every $n, \bar{X}_{n} \bar{Y}_{n}$ has a point in common with $X_{1} Y_{1}$. The point set composed of the point $P$ together with the sum of the $\operatorname{arcs} X_{1} Y_{1}, \bar{X}_{1} \bar{Y}_{1}, \bar{X}_{2} \bar{Y}_{2}, \bar{X}_{3} \bar{Y}_{3}, \cdots$ would then be a connected point set lying within $K$ and containing both $P$ and $X_{1}$. Thus a contradiction would be obtained. Similarly there exists $n_{2}$, greater than $n_{1}$, such that if $n \geqq n_{2}$ then $X_{n} Y_{n}$ has no point in common with $X_{n_{1}} Y_{n_{1}}$. There exists $n_{3}$ greater than $n_{2}$ such that if $n>n_{3}$ then $X_{n} Y_{n}$ has no point in common

* S. Janiszewski, "Sur la géometrie de lignes cantoriennes," Comptes Rendus, vol. 151 (1910), pp. 198-201.

t It may be of interest to note that in order that a bounded continuous set of points $M$ should be a continuous curve it is not sufficient that every two points of $M$ should be the extremities of a simple continuous arc lying wholly in $M$. To see this consider the following example.

Example. Let $A B$ denote the interval from $(0,0)$ to $(0,1)$, in a rectangular system of coordinates, and let $B_{1}, B_{2}, B_{3}, \cdots$ denote the points $(1,1),\left(1, \frac{1}{2}\right),\left(1, \frac{1}{3}\right), \cdots$ respectively. The point set composed of the intervals $A B, A B_{1}, A B_{2}, A B_{3}, \cdots$ is a bounded, continuous set of points $M$ every two points of which can be joined by an arc that lies in $M$. But $M$ is not connected "im kleinen" and is therefore not a continuous curve. However in my paper "A theorem concerning continuous curves," this BULletis, vol. 23 (1917), pp. 233-236, I proved the truth of the converse proposition that every two points of a continuous curve are the extremities of at least one simple continuous arc lying in the point set constituted by that curve. 
with $X_{n_{2}} Y_{n_{2}}$. If this process is continued there will be obtained an infinite sequence of $\operatorname{arcs} X_{n_{1}} Y_{n_{1}} X_{n_{2}} Y_{n_{2}}, X_{n_{8}} Y_{n_{8}}$, ... no two of which have any point in common. For each $i$, the arc $X_{n_{i}} Y_{n_{i}}$ contains, as a subset, an arc $W_{n_{i}} Y_{n_{i}}$ which lies between the circles $\bar{K}$ and $K_{1}$, except for the points $W_{n_{i}}$ and $Y_{n_{i}}$ which lie on $K_{1}$ and $\bar{K}$ respectively. There exist 1 ) on $\bar{K}$ an infinite sequence of distinct points $Y^{\prime}, Y_{1}{ }^{\prime}, Y_{2}{ }^{\prime}, Y_{3}{ }^{\prime}$, $\cdots, 2)$ on $K_{1}$ an infinite sequence of distinct points $W^{\prime}, W_{1}^{\prime}$, $\left.W_{2}{ }^{\prime}, W_{3}{ }^{\prime}, \ldots, 3\right)$ an infinite sequence of distinct $\operatorname{arcs} W_{1}{ }^{\prime} Y_{1}{ }^{\prime}$, $W_{2}^{\prime} Y_{2}{ }^{\prime}, W_{3}{ }^{\prime} Y_{3}{ }^{\prime}, \cdots$ all belonging to the set $W_{n_{1}} Y_{n_{1}}, W_{n_{2}} Y_{n_{2}}$, $W_{n_{8}} Y_{n_{3}}, \cdots$, such that $Y^{\prime}$ is the sequential limit point of the sequence $Y_{1}{ }^{\prime}, Y_{2}{ }^{\prime}, Y_{3}{ }^{\prime}, \ldots$ and $W^{\prime}$ is the sequential limit point of the sequence $W_{1}{ }^{\prime}, W_{2}{ }^{\prime}, W_{3}{ }^{\prime}, \cdots$. No two of the arcs $W_{1}{ }^{\prime} Y_{1}{ }^{\prime}, W_{2}{ }^{\prime} Y_{2}{ }^{\prime}, W_{3}{ }^{\prime} Y_{3}{ }^{\prime}, \cdots$ have a point in common. It easily follows that there exists a closed connected point set $N$, containing $Y^{\prime}$ and $W^{\prime}$, such that every point of $N$ is a limit point of the point set constituted by the sum of the arcs $W_{1}^{\prime} Y_{1}^{\prime}, W_{2}^{\prime} Y_{2}^{\prime}, W_{3}^{\prime} Y_{3}^{\prime}, \cdots$. The point set $N$ is a continuous set of condensation of the set $M$.

Thus the supposition that $M$ is not connected "im kleinen" leads to a contradiction. It follows that $M$ is a continuous curve.

University of Pennsylvania, Philadelphia, Pa.

\section{DERIVATIVELESS CONTINUOUS FUNCTIONS.}

BY PROFESSOR M. B. PORTER.

(Read before the American Mathematical Society October 26, 1918.)

THERE is no more interesting illustration of the refinement of geometric intuition through the influence of the arithmetization of mathematics than that presented by the history of functions of this type. No less a mathematician than Ampère, not to mention Duhamel and Bertrand, thought he had actually proved that continuous functions had derivatives for all save a finite number of arguments. Darboux in his paper on "Discontinuous functions" published in the Annals of the Ecole Normale for 1875, though dated January 20, 1874, in 